\title{
Evaluation des mesures de biosécurité dans les fermes avicoles modernes du district de Bamako dans le cadre de la lutte contre la grippe aviaire
}

\section{Assessment of biosecurity measures in modern poultry farms in the Bamako district as part of the fight against avian influenza}

\author{
Yalcouyé $\mathrm{H}^{{ }^{*}}$, Sangho $\mathrm{F}^{2,3}$, Dara $\mathrm{S}^{1}$, Sangho $\mathrm{O}^{2,3}$, Ouattara $\mathrm{S}^{4}$, Traoré $\mathrm{B}^{5}$, Kayembé Ken ${ }^{5}$, Sangho $\mathrm{A}^{6}$, Coulibaly $\mathrm{CA}^{3}$, Telly $\mathrm{N}^{3}$, Togo $\mathrm{Y}^{7}$, \\ Tounkara $\mathrm{M}^{3}$, NDiaye $\mathrm{HM}^{8}$, Togola $\mathrm{OB}^{9}$, Berthé $\mathrm{M}^{7}$, Doumbia $\mathrm{L}^{3}$, Diallo $\mathrm{B}^{10}$, Diarra $\mathrm{MM}^{11}$
}

1 : Direction Nationale des Services Vétérinaires, Bamako, Mali

2 : Département d'Enseignement et de Recherche des Sciences Biologiques et Médicales, FAPH/USTTB, Mali

3 : Département d'Enseignement et de Recherche en Santé Publique FMOS/USTTB, Mali

4 : Direction Régionale de la Santé de Bamako, Mali

5 : Réseau Africain des Epidémiologiste de Terrain, AFENET-Mali, DGSHP, Bamako, Mali

6 : Département d'Enseignement et de Recherche des Sciences Pharmaceutiques, FAPH / USTTB, Mali

7 : Institut National de Santé Publique (INSP), Bamako, Mal

8 : Direction Régionale de la Santé et de L'Hygiène Publique, Ségou

9 : Centre de Santé de Référence de Tominian, Ségou, Mali

10 : Centre Hospitalier Universitaire Odonto-Stomatologie (CHUCNOS), Bamako, Mali

11 : Centre de Santé de Référence (CSRéf) de Kéniéba, Kayes, Mali

*Auteur correspondant : Dr Hamidou Yalcouyé, Direction Nationale des Services Vétérinaires, Bamako, Mali, 669726 23, 744882 62, yalcouye_sn@yahoo.fr

\section{Résumé :}

Introduction : La biosécurité peut être définie comme « l'ensemble des mesures de nature préventive mises en œuvre pour éviter de contaminer ou d'être contaminé par un agent biologique". Le passage au mode d'élevage intensif pose avec acuité la question de la biosécurité. Les exploitations avicoles modernes sont les plus concernées car la biosécurité, qu'elle soit externe ou interne, impacte la santé en agissant comme facteur préventif de l'introduction ou de la propagation des maladies en élevage. Méthodes : L'administration du questionnaire a été réalisée par méthode téléphonique compte tenue de la distance et de l'insuffisance des ressources. Le travail reposera sur la synthèse des revues bibliographiques, sur l'élaboration du questionnaire et les enquêtes sur le terrain. Le questionnaire a été testé et validé avant son administration sur le terrain. Résultats : Le nombre d'aviculteurs dont la ferme est localisée à Sénou était de $3 / 10$. II est suivi de celui à Gouana avec 2/10. Les informations reçues sur les données de l'exploitation font ressortir que les aviculteurs pratiquant la production d'œufs étaient plus nombreux que ceux produisant la chair. $90 \%$ des aviculteurs interrogés font de la production d'œufs et $10 \%$ de la production de poulet de chair. Les maladies rencontrées dans les poulaillers et qui constituent un problème dans ces fermes sont la maladie de Newcastle pour $90 \%$ et la bronchite infectieuse pour $10 \%$. Conclusion : L'aviculture était plus pratiquée à Sénou et Gouana. Les maladies aviaires les plus fréquentes étaient la Newcastle et la bronchite infectieuse. Les employés ne trempaient pas leurs bottes en accédant aux bâtiments d'élevage.
Mots clés : Evaluation, biosécurité, grippe aviaire, Bamako, Mali

Abstract:

Introduction: Biosecurity can be defined as "the set of preventive measures implemented to avoid contaminating or being contaminated by a biological agent." The switch to intensive farming mode sharply raises the question of biosecurity Modern poultry farms are the most concerned because biosecurity, whether external or internal, impacts health by acting as a preventive factor for the introduction or spread of diseases in farms. Methods: Administration of the questionnaire was carried out by telephone method taking into account the distance and the insufficiency of resources. The work will be based on the synthesis of bibliographic reviews, on the development of the questionnaire and on the field surveys. The questionnaire was tested and validated before its administration in the field. Results: The number of poultry farmers whose farm is located in Sénou was $3 / 10$, followed by that in Gouana with $2 / 10$. training received on farm data revealed that poultry farmers practicing egg production outnumbered those producing meat. $90 \%$ of the poultry farmers surveyed produce eggs and $10 \%$ produce broilers. The diseases encountered in poultry houses and which constitute a problem on these farms are Newcastle disease for $90 \%$ and infectious bronchitis for $10 \%$. Conclusion: Poultry farming was more practiced in Sénou and Gouana. The most common avian diseases were Newcastle and infectious bronchitis. Employees did not soak their boots when entering livestock buildings.

Keywords: Assessment, biosecurity, avian influenza, Bamako, Mali

\section{Introduction}

Le Mali est un pays à vocation essentiellement agropastorale. La biosécurité peut être définie comme " l'ensemble des mesures de nature préventive mises en œuvre pour éviter de contaminer ou d'être contaminé par un agent biologique » $(1,2)$. Selon certains auteurs, il est difficile de trouver une définition de la biosécurité, ce terme pouvant être utilisé dans plusieurs domaines d'activités (3). Dans le domaine de la santé animale, la définition de la biosécurité peut être très large avec cinq composantes (3):

- Bio-exclusion : le pathogène ne rentre pas dans un troupeau ; 
- Bio-compartimentation : le pathogène ne circule pas dans un troupeau ;

- Bio-confinement : le pathogène ne sort pas d'un troupeau

- Bio-prévention : le pathogène n'infecte pas l'homme ;

- Bio-contamination : le pathogène ne persiste pas dans l'environnement.

Dans un contexte de concurrence internationale accrue, les productions hors sol se sont multipliées et les élevages se sont agrandis et densifiés ; des régions ont spécialisé leurs productions (4).

Le passage au mode d'élevage intensif pose avec acuité la question de la biosécurité. Les exploitations avicoles modernes sont les plus concernées car la biosécurité, qu'elle soit externe ou interne, impacte la santé en agissant comme facteur préventif de l'introduction ou de la propagation des maladies en élevage (3). Si l'élevage participe pour $10 \%$ à la formation du PIB national (5), le secteur de l'aviculture constitue sa branche la plus dynamique.

La production avicole nationale est concentrée dans la région de Koulikoro autour du District de Bamako. Cette région assure $69,9 \%$ de la production avicole moderne totale du Mali contre 26,4\% du District de Bamako (6). En réalité, les fermes de Koulikoro sont tenues par des citadins de Bamako (6).

À la ferme, les mesures de biosécurité visent à prévenir la contamination du site par des pathogènes (bio exclusion), à éviter la propagation d'un pathogène déjà présent entre différents groupes d'animaux (bio gestion), et à empêcher que ce pathogène puisse se propager à d'autres fermes (bio confinement) (7).

L'élevage avicole est un secteur qui se développe de plus en plus dans tous les pays en voie de développement. Cette tendance à mettre en place des animaux à la limite de leurs capacités physiologiques s'accompagne bien évidemment d'une fragilité exacerbée envers les divers agents pathogènes présents dans leur environnement. Qu'il s'agisse de microorganismes commensaux des élevages ou d'agents pathogènes plus spécifiques, leur présence et surtout la pression infectieuse qu'ils exercent ont pour conséquence une dégradation des performances des animaux, une augmentation de la fréquence et de la gravité des maladies, ainsi qu'un accroissement des frais vétérinaires (8). Tous ces éléments vont entraîner une perte de production, une baisse des revenus de l'éleveur et une dégradation des produits issus de ces élevages constituant ainsi une menace pour la santé publique. II convient donc de mettre en place des mesures de prévention vis-à-vis de ces divers agents pathogènes, très développés en élevage aviaire. II conviendra donc de se pencher davantage sur des considérations zootechniques et épidémiologiques usuelles, des plans de médication préventive ou encore des protocoles de désinfection de locaux ou de matériels (8).
La proximité d'élevages infectés revient également et fréquemment comme facteur de risque dans plusieurs études menées en Angleterre, en Irlande du Nord suggérant soit la transmission directe ou indirecte d'un élevage infecté à un élevage sain voisin, soit la présence d'une source de contamination commune (9).

Nous rencontrons principalement deux formes d'aviculture : l'aviculture traditionnelle qui exploite des sujets locaux et l'aviculture moderne qui utilise des races exotiques à potentiel génétique élevé. Cette deuxième forme d'aviculture est plus pratiquée dans les zones périurbaines de Bamako, dans les capitales régionales et dans le District de Bamako. Elle bénéficie de la subvention sur le budget d'Etat pour augmenter la productivité, le revenu des professionnels de la filière et de créer des emplois massifs par l'installation des jeunes et des femmes dans l'activité d'aviculture (10) mais aussi d'améliorer la qualité nutritionnelle des produits avicoles (chair et œufs).

Toutes ces raisons, associées à l'insuffisance d'études et de données sur le sujet, ont prévalu au choix du thème de cette étude. L'objectif général était d'évaluer le niveau d'application des mesures de biosécurité dans les fermes avicoles.

\section{Méthodes \\ Cadre de l'étude}

Le milieu d'étude était le District de Bamako dans son ensemble (six communes en zone urbaine) (carte 1). Située sur les rives du fleuve Niger, appelé « Djoliba », la ville de Bamako est construite dans une cuvette entourée de collines. Elle s'étend d'ouest en est sur $22 \mathrm{~km}$ et du nord au sud sur $12 \mathrm{~km}$, pour une superficie de $267 \mathrm{~km}^{2}$ (11). Le District de Bamako compte 2446700 habitants? en 2018. Son rythme de croissance urbaine est actuellement le plus élevé d'Afrique et la capitale Bamako est érigée en district et divisée en six communes. Bamako occupe la frange la plus méridionale du Sahel africain correspondant à la zone soudanienne. Elle bénéficie de ce fait d'un climat tropical assez humide avec un total des précipitations annuelles de 878 millimètres mais avec une saison sèche et une saison des pluies bien marquées.

\section{Type d'étude}

Nous avons mené une étude transversale descriptive.

Période d'étude

L'étude s'est déroulée du 20 octobre 2019 au 11 novembre 2019.

\section{Population d'étude}

La population d'étude était constituée par les aviculteurs du district de Bamako.

\section{Echantillonnage}

II y avait cinquante-quatre fermes avicoles dans la circonscription administrative de Bamako. Notre étude a porté sur dix (10) aviculteurs. Cet échantillon représente $18,51 \%$ de l'échantillon total. Nous avons procédé au tirage aléatoire systématique de 10 aviculteurs sur les 54 que comptait la base de sondage. Cette base des 
aviculteurs du Mali nous a été fournie par la Direction Nationale des Productions et des Industries Animales (DNPIA).

\section{Critères d'inclusion}

Les critères d'inclusion étaient :

- $\quad$ Etre aviculteurs faisant l'élevage intensif ;

- $\quad$ Etre dans la zone d'étude.

Critères de non inclusion

Les critères de non inclusion étaient :

- $\quad$ Refuser de participer à l'étude ;

- $\quad$ Etre indisponible pour participer à l'étude.

Outils et techniques de collecte des données

L'administration du questionnaire a été réalisée par méthode téléphonique compte tenu de la distance et de l'insuffisance des ressources.

Le travail a consisté en une revue bibliographique, l'élaboration du questionnaire et les enquêtes sur le terrain. Le questionnaire a été testé et validé avant son administration sur le terrain.

\section{Traitement et analyse des données}

Les fiches d'enquête ont été saisies sur Excel 2013. Les données ont été nettoyées et appurées.

Les données ont été analysées sur Epi Info7. La moyenne, la fréquence et le pourcentage des aviculteurs qui adoptaient les mesures de biosécurité ont été calculés. Considérations éthiques

Dans le cadre de cette étude, aucun contact n'a été fait directement avec les aviculteurs. En aucun cas leur utilisation ne permet de révéler l'identité des propriétaires des fermes avicoles. Le travail a été donc fait dans un contexte d'anonymat complet.

\section{RESULTATS}

Le nombre d'aviculteurs dont la ferme est localisée à Sénou était de $3 / 10$. II est suivi de celui à Gouana avec 2/10 (tableau I).

Les informations reçues sur les données de l'exploitation faisaient ressortir que les aviculteurs pratiquant la production d'œufs étaient plus nombreux que ceux produisant la chair. $90 \%$ des aviculteurs interrogés font de la production des œufs et $10 \%$ de la production de poulet de chair (figure 1)

L'évaluation des questions relatives aux mesures sanitaires de l'exploitation montrait que 9/14 de ces questions avaient été répondues à l'affirmative, soit $64 \%$ de oui. Deux questions enregistraient des réponses négatives, soit $14 \%$ (figure 2).

Pour la désinfection des poulaillers, les deux produits majoritairement utilisés par les aviculteurs étaient le vircon $(n=5)$ et le virunet $(n=5)$

Les maladies rencontrées dans les poulaillers et qui constituent un problème dans ces fermes sont la maladie de Newcastle pour $90 \%$ et la bronchite infectieuse pour $10 \%$.

Les $100 \%$ du personnel employé possèdaient des tenues de travail et se désinfectaient avant toute opération. Cependant, $100 \%$ de ce personnel de travail ne trempent pas leurs bottes dans le pédiluve en accédant dans poulaillers (figure 3 ).

\section{DISCUSSION}

Le choix de la zone d'étude se justifiait par le fait que la majorité des productions avicoles étaient concentrées dans les localités périurbaines de Bamako.

Le manque d'informations sur la liste et la localisation des fermes nous avaient conduit à utiliser une méthode d'échantillonnage systématique.

Les résultats de l'étude sur le respect des mesures de biosécurité avaient montré que l'aviculture était plus pratiquée dans les zones de Sénou et de Gouana. Cela s'expliquerait par l'existence d'espace convenable à la pratique de l'aviculture.

L'analyse des mesures sanitaires et hygiéniques faisait ressortir que :

- la durée du vide sanitaire était respectée par l'ensemble des aviculteurs enquêtés soit $100 \%$. Ce résultat est similaire à ceux trouvés par DOSSO $\mathrm{S}$. (13) et TRAORE AB (12) ;

- $100 \%$ des aviculteurs ont répondu que les animaux étaient sains ;

- L'analyse du système d'abreuvement a montré que l'eau servie aux animaux était potable. Cette eau provenait des forages ou des puits à grand diamètre. Ce résultat est similaire à celui obtenu par TRAORE $\mathrm{AB}(12)$;

- Les aliments servis aux animaux étaient bien conservés pour $90 \%$ des aviculteurs. $10 \%$ des aviculteurs ont évoqué des difficultés de conservation des aliments ;

- L'analyse de la propreté des abreuvoirs et des mangeoires avait trouvé que $50 \%$ des aviculteurs respectaient le nettoyage après chaque utilisation et $50 \%$ avaient reconnu qu'ils n'étaient pas réguliers dans le nettoyage de ces matériels ;

- Le vide sanitaire après chaque bande était pratiqué par tous les aviculteurs, soit $100 \%$ de réponse positive ;

- Les oiseaux morts étaient plutôt brûlés par tous les aviculteurs. Les aviculteurs disent ne pas posséder d'incinérateur ;

- $100 \%$ des aviculteurs disaient que leurs fermes ne sont pas situées près d'une route principale ;

- L'analyse du voisinage avec d'autres fermes avait trouvé que $50 \%$ ont des voisinages avec d'autres fermes avicoles tandis que 50\% n'ont pas de voisinage ;

- $100 \%$ des aviculteurs avaient répondu qu'il n'y avait pas un libre accès à leurs fermes ;

- L'analyse des désinfectants utilisés a trouvé que $50 \%$ des aviculteurs utilisent le vircon tandis que $50 \%$ utilisent le virunet ;

- L'analyse des maladies fréquentes a trouvé que $90 \%$ des aviculteurs signalaient la maladie de Newcastle tandis que $10 \%$ signalaient les maladies respiratoires comme la bronchite infectieuse. 
L'analyse de la conduite du personnel a trouvé que 100\% des employés possédaient des tenues de travail et se désinfectaient avant toute opération dans le poulailler, tandis que $100 \%$ de ces employés ne trempaient pas les bottes dans le pédiluve. Cela nous a fait penser que même si le dispositif du pédiluve existe, ce dernier ne contient pas la solution pour désinfecter. Ce résultat est similaire à celui trouvé par Amadou dit Baba TRAORE (12) et Babacar N (14).

\section{Conclusion}

L'aviculture était plus pratiquée dans les zones de Sénou et de Gouana. Dans 100\%, les aviculteurs possédaient des animaux sains, servaient de l'eau potable aux animaux, pratiquaient le système de bande unique et éliminaient les oiseaux malades. Les maladies fréquentes étaient la maladie de newcastle et la bronchite infectieuse. II n'y avait pas un libre accès dans $100 \%$ des fermes des aviculteurs enquêtés. Aucun des employés ne trempait ses bottes dans le pédiluve en accédant au poulailler. II a été recommandé de respecter les mesures de biosécurité dans les fermes avicoles ; de nettoyer et désinfecter les matériels d'élevage après chaque utilisation.

\section{Remerciements}

- Gouvernement malien (Ministère de la santé et des Affaires Sociales, Ministère de l'Elevage et de la Pêche, Ministère de l'Environnement).

- CDC/AFENET pour le financement de cette activité.

- Formateurs et mentors pour leur accompagnement.

- USTTB, DER de Santé Publique.

\section{Références}

1. Véron $L$, Cavalerie $L$, Chevalier $F$, Jabert $P$, Tourette I, Bronner A. BIOSECURITE EN ELEVAGE BOVIN VIS-A-VIS DE LA TUBERCULOSE EN FRANCE : REVUE DES FACTEURS DE RISQUE DE CONTAMINATION DES ELEVAGES ET DES MESURES DE PREVENTION DISPONIBLES. :15.

2. Calvar C, Lemoine T. EN ELEVAGE DE PRODUCTION. 2014;12.
3. Corrégé I. La biosécurité en élevage de porcs : enjeux, observance, freins et perspectives de progrès. $: 12$.

4. Guériaux D, Fédiaevsky A, Ferreira B. La biosécurité : investissement d'avenir pour les élevages français. Bul de l'Ac Vét de France. 2017;(1):112.

$5 . \quad$ Filière volaille : SEDIMA-MALI forme les aviculteurs - Bamada.net [Internet]. [cité 29 oct 2019]. Disponible sur: http://bamada.net/filiere-volaille-sedimamali-forme-les-aviculteurs

6 . Étude de marché de la filière volaille PACEPEP_2015. :90.

7. 5783a99608ae3f355b4a1c76.pdf [Internet] [cité 23 oct 2019]. Disponible sur:

https://www.researchgate.net/profile/Manon_Racicot/publ ication/305158030_Biosecurite_Principes_et_points_criti ques/links/5783a99608ae3f355b4a1c76.pdf

8. Selma O, Alloui N. Biosécurité en Aviculture Biosecurity in Poultry Production. :96.

9. Alloui N, Ayachi A. BIOSECURITY PRACTICES IN ALGERIAN POULTRY FARMS. :5.

10. Mali: Aviculture traditionnelle - Le ministre de l'élevage arme les femmes [Internet]. allAfrica.fr. 2018 [cité 29 oct 2019]. Disponible sur: https://fr.allafrica.com/stories/201803090389.html 11. Bamako. In: Wikipédia [Internet]. 2019 [cité 1 nov 2019]. Disponible sur:

https://fr.wikipedia.org/w/index.php?title=Bamako\&oldid= 163992439

12. TRAORE A dit B. Evaluation des pratiques avicoles et de l'antibiorésistance des salmonelles isolés chez les poules pondeuses en zone péri urbaine de Bamako. EISMV-Dakar; 2016.

13. Dosso. Analyse des pratiques avicoles et de l'usage des antibiotiques en aviculture moderne dans le departement d'agnibilekrou (Côte d'Ivoire). EISMVDakar; 2014.

14. Babacar N. Essai d'amélioration des performances de croissance de poulet de chair dans la région périurbaine de Dakar par application des bonnes pratiques techniques et sanitaires de la charte sanitaire " SENEVOL ". : :98. 
Liste des tableaux et figures

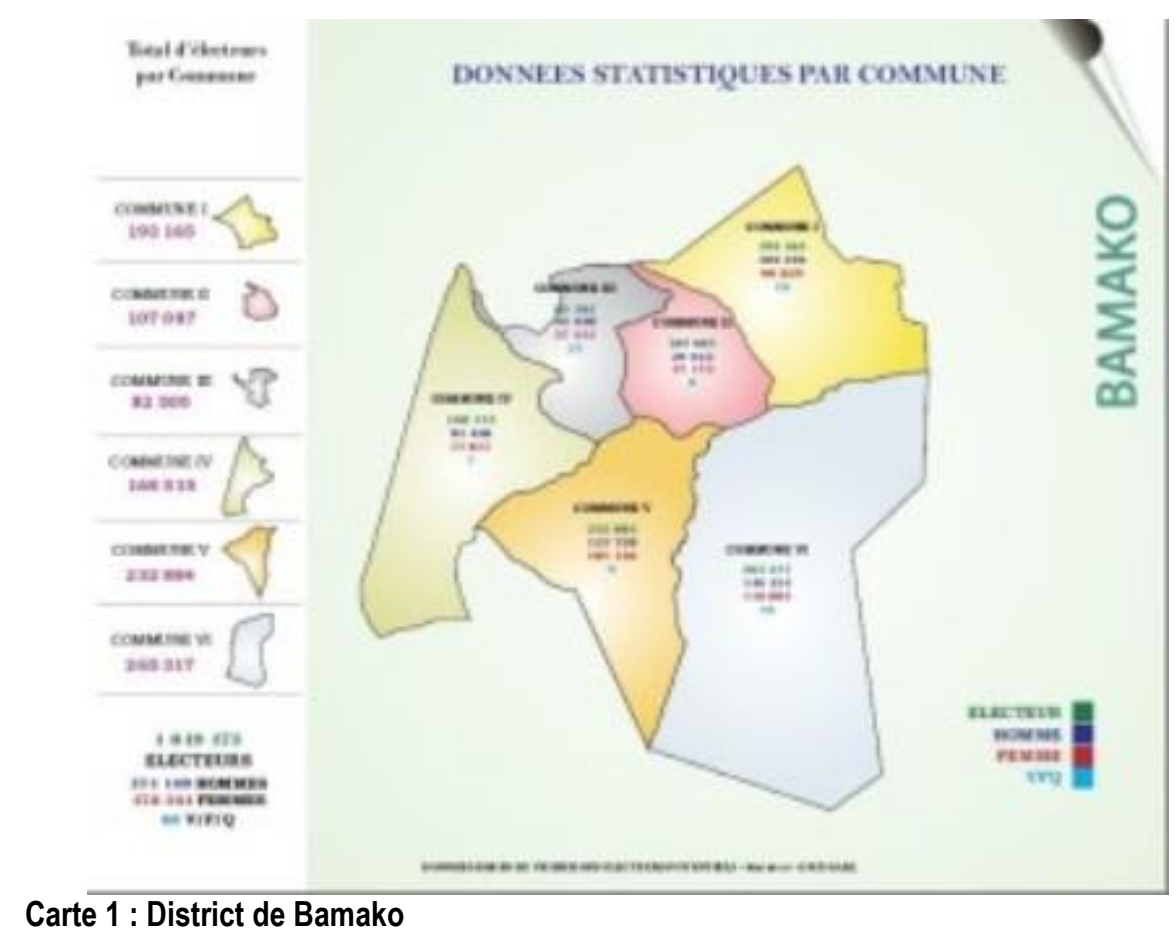

Tableau III : Localisation des fermes par site

\begin{tabular}{lc}
\hline \hline Sites & Nombre de fermes \\
\hline \hline Fombabougou & 1 \\
Gouana & 2 \\
Kabala & 1 \\
Missabougou & 1 \\
Sébénicoro & 1 \\
Sénou & 3 \\
Titibougou & 1 \\
Total & 10 \\
\hline \hline
\end{tabular}




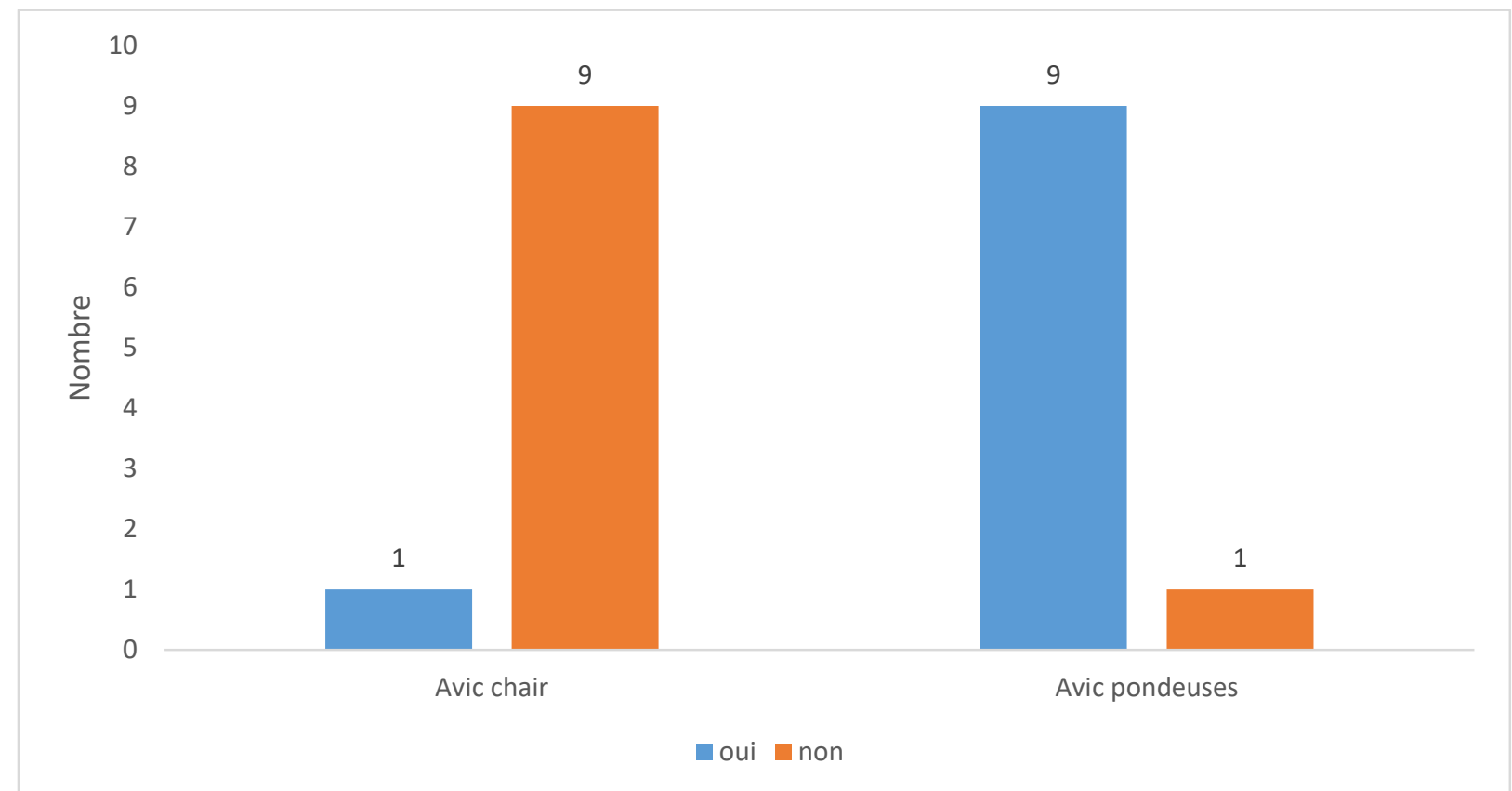

Figure 4 : Nombre d'aviculteurs pour pondeuses et poulets de chair

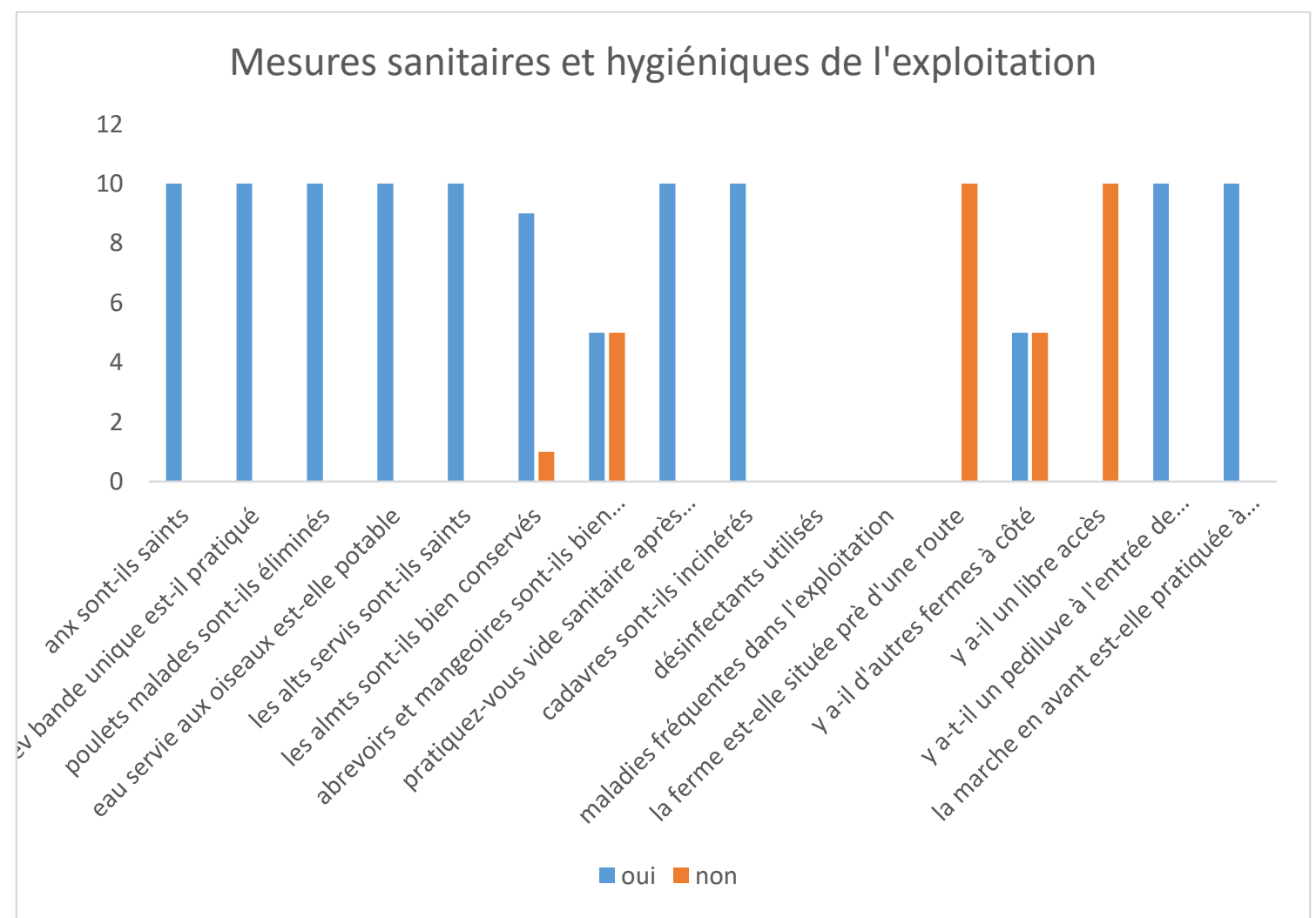

Figure 5 Mesures sanitaires et hygiène de l'exploitation 
Conduite du personnel

12

10

6

4

2

0

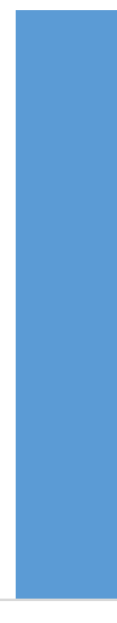

le personnel a-il tenues travail individuel

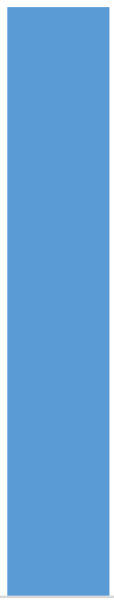

personnel se désinfete-il avant personnel trempe-t-il les bottesdans toute opération dans le poulailler le pediluve avant toute opération

oui non

Figure 6 : Conduite du personnel 